\title{
Testo Tempo Verità
}

\section{Domenico Fiormonte \\ Università Roma Tre}

Abstract. This article tries to show the strict interdependence of the concepts of text, time, and truth in relation to textual transmission. It develops the thesis that the identity of the text is a function of a series of actors working on the historic, cultural, religious, and other levels. It offers the example of the origination of the Old Testament, which is considered the real foundational act of Western practices of identity construction/reconstruction. This event generated the metaphysics of the text that produced the overlapping of theological and philological truth. Nonetheless, the digital dimension seems potentially able to call into crisis the pact of identity that is based on the assumed stability of the written document and the idea of time that derives from it. The present scenario sees a tension between the data preservation/retrieval paradigm and the creative/performative style of online writing. We are facing a dialogue and interaction with and between machines that the methods and traditional tools of the humanist sciences find increasingly difficult to understand, describe, and map.

In questo articolo si cerca di mostrare la stretta interdipendenza dei concetti di testo, tempo e verità in relazione alla storia della trasmissione dei testi. Viene avanzata la tesi che l'identità del testo sia funzione di una serie di attori che agiscono sul piano storico, culturale, religioso, ecc., e a tale proposito viene portato l'esempio della nascita dell'Antico Testamento, vero atto fondativo delle pratiche occidentali di costruzione/ricostruzione dell'identità. Dalla metafisica del testo generata da questo evento scaturisce la sovrapposizione di verità teologica e verità filologica. Ma la dimensione digitale sembra potenzialmente in grado di mettere in crisi il patto identitario fondato sulla presunta stabilità del documento scritto e sulla concezione tempo che ne deriva. Lo scenario presente vede una tensione fra il paradigma della conservazione/retrieval dei dati e quello produttivo-performativo delle scritture online. Un dialogo e un'interazione con $e$ fra le macchine che i metodi e gli strumenti tradizionali delle scienze umanistiche fanno sempre più fatica a comprendere, descrivere, mappare.

[Nisargadatta] Maharaj said quite often that books get written; they are never written by authors. Only a little thought is necessary to see the truth of what he meant. He was NOT referring only to books on spiritual matters; he was referring to 
all books. In the overall functioning of the manifested universe, whatever was necessary as written or spoken words appeared spontaneously ... No credit or blame could attach to any individual writer for the simple reason that the individual is a mere illusion and has not autonomous existence.

Ramesh S. Balsekar, Experience of Immortality.

E varrebbe la pena di ripercorrere la storia dell'edizione dei testi in rapporto con le diverse concezioni di verità, e perciò dell'autorità da conferire ai testi stessi. Qui n è possibile.

Cesare Segre, Avviamento all'analisi del testo letterario.

\section{Introduzione: Tre giganti incatenati}

Pubblico per la prima volta in italiano questo contributo che - in omaggio alla mobilità che dà il titolo al nostro volume - ha subito nel tempo una serie di modificazioni1. Mi accingo a connettere insieme tre parole il cui suono fa già tremare i polsi: Testo tempo verità.

Che cosa unisce, almeno nella mia prospettiva, questi tre giganti incatenati (e non solo perché privi di interpunzione delimitatoria)? Quello che cercherò di abbozzare è l'origine e l'ubi consistam della concezione universalista e monologica del testo. Ovviamente è sopra (e sotto) il testo che troviamo il tempo, senza il quale non esiste né testo né verità. Mi limiterò a illustrare il legame fra verità testuale e relativa concezione del tempo attraverso l'uso di alcune "scene," rinunciando a sostenere un'argomentazione rigorosa, se non nel senso che gli attribuisce Olivier Reboul, quando definisce l'argomentazione un metodo a metà strada “fra il necessario e l'arbitrario" (Reboul 102). La mancanza qui e lì di nessi, qualche interpolazione (vedi esergo) e la presenza di ambiguità espositive, testimoniano che questo lavoro è e non può che rimanere precario, programmaticamente instabile e aperto.

\section{Assalto alla maestà}

Manuali e dizionari in genere ci forniscono tre classi di definizioni del - o informazioni sul - testo:

- Un'etimologia: la parola deriva dal latino "textus" che significa tessuto. Si tratta quindi di qualcosa che sta insieme, le cui parti sono strettamente intrecciate tra loro;

- Un'ipotesi: il testo è anche ciò che viene prodotto e scambiato in una comunicazione linguistica;

- Una definizione: il testo è una produzione linguistica, scritta o orale, realizzata con l'intenzione e l'effetto di comunicare qualcosa a qualcuno.

Sin qui nulla che richiami direttamente la nozione di "verità" (indugerò molto in questo intervento sull'ambiguità fra "verità testuale" e "testo veritiero," poiché le due espressioni sono forse logicamente, ma non storicamente distanti). Il problema della "verità" del testo 
infatti viene introdotto a un certo punto della storia, quando la scrittura comincia a solidificarsi in prodotti stabili - o almeno percepiti come tali (Jaynes 218-249). Il testo cioè non è più un supporto della comunicazione orale o una funzione di contesti sociali, religiosi, ecc., ma acquista una sua indipendenza. Come sappiamo questa "indipendenza," che è la forza del testo, rappresenta per Platone insieme un pericolo e il suo limite più grande: e infatti egli attacca "il discorso muto" della scrittura contrapponendolo alla parola viva (la "verità" si raggiunge solo dialetticamente).

Ma quando accade che la verità smette di essere dialettica e viene consegnata al testo? Una riflessione che affonda le sue radici negli anni Trenta del secolo scorso (da Walter Benjamin a Paul Valèry), e che culmina negli anni Cinquanta e Sessanta (da Harold Innis a Marshall McLuhan) è concorde nell'identificare lo spartiacque nell'invenzione e diffusione della stampa. Lo stesso concetto di "autore," che si svilupperà e culminerà nel romanticismo (Woodmansee 35-55), nasce grazie alla diffusione in più copie identiche dello stesso oggetto-libro. Insomma riproducibilità, oggi come ieri, è sinonimo di identità (Mordenti). Tuttavia la nozione di verità del testo, nel senso più puramente teo-filologico, sembra essere assai più antica e soprattutto più legata alle radici della nostra cultura:

Ora, o re, emana il decreto e fallo mettere per iscritto, perché sia irrevocabile, come sono le leggi di Media e di Persia che non si possono mutare. (Daniele, 6, 9) ${ }^{2}$

I satrapi del regno di Dario sono invidiosi di Daniele, prediletto del re e promosso ad alti incarichi. L'unico modo per incastrarlo è colpirlo nella pratica della fede: pretesto per l'accusa diventerà la sua preghiera rituale nel momento in cui si chiede che essa diventi illegale. È perciò che i nemici di Daniele convincono il Re babilonese a emanare un decreto in cui si stabilisce che chiunque "rivolga supplica alcuna a dio o uomo all'infuori del re" deve essere gettato nella fossa dei leoni, ma soprattutto sanciscono che debba essere firmato perché diventi "irrevocabile."

Se questa è una delle testimonianze più belle della fondazione della "stabilità" del testo, l'episodio ne testimonia tutta l'ambiguità e la potenza contraddittoria. Il potere qui si incarna nel Testo "pubblicato" che diventa sinonimo di "immutabile." Ma questo stesso potere è vittima del Testo, cioè di un sé scagliato su un supporto: il Re Dario, che pure ama e rispetta Daniele, sarà costretto a far eseguire il decreto che ha lui stesso promulgato. La maestà del Testo è superiore alla maestà della persona. Anche il più potente dei Re, di fronte al Testo, si deve inchinare. La parola, lontana da chi l'ha proferita, è una condanna. Ma anche una garanzia per chi l'ascolta.

La lezione dei nemici di Daniele, tuttavia, servì allo scopo. È proprio al tempo della cattività babilonese (qui reale, là mitema) infatti che gli Ebrei elaborano il loro Testo. La cui maestà sarà destinata ad avere effetti ben più profondi di una condanna a morte.

Mario Liverani, nel suo Antico Oriente. Storia, società, economia, sintetizza la nota questione della "composizione" dell'Antico Testamento. Si tratta di una vicenda assai complessa che riassumerò per sommi capi (lo stesso Liverani la narra in modo più esteso in Oltre la Bibbia). In sostanza, in seguito alle invasioni assiro-babilonesi del Regno di Israele 
(VIII-VI sec. a.c.), avvengono successive ondate di deportazioni delle élites giudee nei territori dei conquistatori:

Nabucodonosor espugna Gerusalemme una prima volta, riducendola alla condizione di regno vassallo (597), e poi una seconda volta ponendo fino all'autonomia locale (586). ... E mentre i deportati in altre zone assire venivano fusi (anche forzosamente) alle popolazioni locali, gli esuli giudei in Babilonia mantennero maggiore coesione e individualità.” (Liverani, Antico Oriente 680-681)

Dunque assistiamo a un doppio movimento di popolazioni, deportati in arrivo, che “portarono le loro divinità e le loro costumanze" in Palestina (682) ed élites palatine o templari che cercano di perpetuare nell'esilio babilonese "la purezza della loro lingua, delle loro costumanze, della loro religione, per difendersi dall'assimilazione” (682). Ivi compresi i testi sacri. A questo punto però avviene qualcosa di singolare, ovvero gli esiliati tornando nella loro terra notano che le antiche tradizioni sono scomparse o si sono corrotte, insomma non la "riconoscono". E cominciano a considerarsi, in quanto scampati al disastro nazionale, gli unici eredi legittimi. Essi "restaurano tempio e legge, vietano matrimoni misti e sincretismo religioso, considerano illegittima la presenza sul territorio di coloro che non fanno parte della comunità religiosa yahwistica..." (687). È a partire da questo momento che inizia quella che Liverani chiama la "rifondazione storiografica", ovvero l'operazione di riscrittura della storia di Israele in chiave nazional-religiosa:

Ricollocando alle epoche di redazione i singoli interventi testuali che assommati insieme costituiscono l'Antico Testamento, ci si accorge che la maggior parte di essi si colloca ... in epoca post-esilica (dunque in età achemenide ed ellenistica) - e in misura minore proprio ai limiti estremi di essa, in età esilica. Certamente il complesso dell'Antico Testamento è da valutarsi e da apprezzarsi più nella prospettiva del secondo tempio che non del primo, più nel quadro del ritorno dall'esilio che non della formazione e dello sviluppo del regno di Israele, e costituisce un caso colossale di ripensamento della storia passata, e di una sua riscrittura, in funzione del presente (un presente assai posteriore alla storia narrata) (Liverani, Antico Oriente 690).

Siamo insomma di fronte a un caso, forse il più eclatante, in cui l'identità del testo è funzione del suo contesto culturale (religioso in questo caso). Più di due millenni prima di Google assistiamo a un episodio di manipolazione retroattiva delle forme di (auto) rappresentazione della realtà - i testi - che avrà conseguenze inimmaginabili nella storia dell'Occidente. Il tempo del testo diviene il tempo dell'uomo.

\section{I rinnegati del testo}

Il consolidarsi e rapprendersi dei prodotti dello spirito su un supporto materiale che i poteri non sono estranei nel rendere sempre più stabile e sempre più fisso (Innis), produce quella rivoluzione della conoscenza nella quale siamo ancora immersi. Vari secoli dopo la sua invenzione e diffusione, il testo, può essere così definito da un critico e filologo: 
Se consideriamo i segni grafici (lettere, interpunzione, ecc.) come significanti di suoni, pause, ecc., e riflettiamo sul fatto che questi segni possono essere trascritti più volte e in vari modi (per esempio con grafia e caratteri diversi), restandone immutato il valore, possiamo concludere che il testo è l'invariante, la successione di valori, rispetto alle variabili dei caratteri, della scrittura, ecc. ... Il testo è dunque una successione fissa di segni grafici. Questi significati grafici sono poi portatori di significati semantici ...; ma occorre insistere in partenza su questa costituzione originaria. Occorre insistervi, perché le ricchissime, praticamente infinite implicazioni di un testo, quelle che richiamano lettori ai testi anche per secoli e millenni, sono tutte racchiuse nella letteralità dei significati grafici. Di qui l'importanza della filologia, che s'impegna nella conservazione il più possibile esatta di questi significati. Il fatto che la sopravvivenza dei testi implichi inevitabili guasti nella loro trasmissione deve sollecitare ancor più lo sforzo di tutelarne la genuinità. (Segre, Avviamento 29-30; corsivi miei). ${ }^{3}$

Tralasciamo per un momento alcune lacune interne di questa lunga definizione (non si fa accenno, almeno qui, al ruolo autonomo della forma del testo, né alla sua dimensione pragmatica) e concentriamoci su tre parole chiave: testo come invariante, sua conservazione e genuinità. Non siamo ancora alla "verità" ma ci stiamo avvicinando (e non solo per allitterazione).

Immagino che per alcuni apparirà persino troppo scontato (oggi 2012 ma la prima edizione dell' Avviamento è degli anni Ottanta) che una parola come 'invariante' riferita a un testo rimandi a un mondo sepolto - per analogia e contrasto vengono subito in mente le "scritture mutanti" della rete. Restando alla definizione, considerare i segni grafici delle varianti che rimandano a un "valore immutato" vuol dire proiettare il testo in un iperuranio senza tempo; e d'altronde se, come viene ammesso, il testo può variare, allora com'è possibile che il suo valore possa rimanere immutato nel tempo? Da dove verrebbe attinta questa sua intangibilità?

Tuttavia trattare con sufficienza questa definizione sarebbe avventato - e soprattutto pericoloso. Perché, in realtà, siamo di fronte all'unica concezione del testo riconosciuta come tale dalla disciplina che al testo ha dedicato immensi sforzi teorici e applicativi, ottenendo i più grandi e duraturi risultati: la filologia. E questa disciplina, regina delle discipline storiche e vanto delle scienze umanistiche, per quanto acciaccata e in crisi, è ancora oggi, a più di un secolo dagli strali di Nietzsche, la dominatrice e custode dei metodi di rappresentazione, produzione e gestione della conoscenza scritta. Cioè, almeno per la civiltà occidentale, della conoscenza tout court. Con tutto ciò che consegue a livello sociale, politico, economico, culturale: leggi e carte costituzionali, contratti di matrimonio e polizze sulla vita, computer e giornali, reti autostradali e civiltà urbane. Queste conquiste - se così vogliamo chiamarle - sorgono dal patto fondato sul documento scritto, il corpo-monumento, che viene inciso, scolpito, reso pubblico, ma raramente vissuto.

Si comprende dunque perché sempre Segre, che pure venti anni prima aveva definito il testo come "un concetto limite,” (Segre, Avviamento 362) scrive nel 2001: 
Il testo è tutto il nostro bene; nessuna nostra escogitazione per quanto brillante o suggestiva può valere e significare di più del testo nella sua maestà. Questa maestà coincide con la verità, che è nostro dovere perseguire con impegno, nel testo e ovunque. Potrebbe essere questo il primo comandamento in una specie di giuramento di Ippocrate dei critici letterari. $\mathrm{E}$ non $\mathrm{mi}$ dispiace che nell'imperversare irrefrenabile dei mass media, nel trionfo della virtualità, nell'assordante sovrapposizione di voci e parole ormai dissanguate del loro senso, ci siano discipline che contengano un insegnamento, oltre che metodologico, anche deontologico. (Segre, Ritorno alla critica 99)

Ecco è apparsa "la verità". Però non riluce, non illumina e sembra anzi piuttosto minacciosa. Il testo diventa il solo orizzonte etico, anzi si incarna e si identifica con l'etica stessa. Siamo ben oltre la ricostruzione della "verità testuale”. Chi è fuori dal testo, non solo non possiede la verità (letterale e a questo punto spirituale del testo), ma è bandito dal consorzio umano. È un rinnegato. È questa l'inevitabile parabola della religione del testo: imbastire alla contemporaneità il processo per eresia.

Luciano Canfora ha dedicato un pamphlet al problema della condanna della critica testuale da parte della chiesa cattolica (ma problemi analoghi si riscontrano in altre confessioni), legando i destini della filologia alla possibilità stessa di esercitare il pensiero critico. Titolo e sottotitolo del saggio sono già una tesi: Filologia e libertà. La più eversiva delle discipline, l'indipendenza di pensiero e il diritto alla verità. Ma anche in questo caso si parla di verità: certo si tratta di una verità costruita, almeno idealmente, nel confronto libero delle opinioni e attraverso studi rigorosi, storicamente fondati. Alla fine però il rischio è che si tratti di un atto di fede ontologicamente affine; come nell'Antico Testamento, terreno di lotta fra due verità, quella critico-testuale e quella teologica (Barbaglia, "La rilevanza ermeneutica”; Prato, “Gli scritti biblici”).

Sarebbe ingiusto negare ai filologi il desiderio di libertà o attribuirgli un eccesso di conservatorismo. Ciò di cui parlano Segre e Canfora è naturalmente l'accettabilità e l'accertabilità di un testo. E accettabilità non vuol dire verità. Tuttavia l'accettabilità presuppone un circuito che a sua volta comporta l'introduzione di gerarchie, sistemi di valutazione (la falsificabilità dell'esperimento) e in definitiva giudizi. E la filologia in fondo non è altro che una teoria del giudizio. Dunque il problema della verità teologica, uscito dalla porta, rientra dalla finestra: chi stabilisce, infatti, le gerarchie? Chi dovrebbe garantire la democraticità e scientificità dei metodi, se non un'altra comunità, che nel corso tempo si potrà costituire inesorabilmente come casta? Il metodo, il percorso, non è mai neutrale. E persecutori e perseguitati alla fine si ritrovano intrappolati nello stesso labirinto seriale.

\section{Tempus digitale}

A dire il vero, anche all'interno della filologia, qualcuno aveva iniziato a reagire all'orizzonte apocalittico prospettato poco sopra. La filologia della "frattura e degli scarti", invocata da Corrado Bologna, e il rispetto per la "verità dei fruitori", di cui parla Roberto Antonelli ("Spazio, tempo e testualità"), sono un onesto tentativo di salvare il salvabile, 
ovvero la varietà storica del documento, legata al contesto dinamico della cultura in cui quel documento nasce e si "sviluppa". Grazie a questi e altri studiosi ${ }^{4}$ è emersa, attraverso uno studio più attento dei supporti e degli elementi visuali e materiali della scrittura (Storey), una concezione dinamica del testo, che non viene più solo considerato come un prodotto, ma come un processo. Partendo da considerazioni simili, ma confrontandosi più esplicitamente con l'antropologia culturale, Benozzo propone di fondare un'etnofilologia, ovvero lo studio dell'insieme dei processi e dei contesti che conducono alla fissazione dell'artefatto culturale (35-63). Credo non sia sbagliato ipotizzare che la comparsa del supporto digitale abbia fornito un potente stimolo a queste riflessioni. E il problema del "riconoscimento e della costruzione" del dato (Bologna 50), nell'incontro con l'informatica, diventa l'atto stesso di fondazione di una nuova disciplina neo-(post?)filologica: l'informatica umanistica. Di fronte alla constatazione che l'informatica implica un ripensamento del nostro rapporto col dato, e di conseguenza del nostro modo di fare ricerca, è chiaro che il progetto di questa disciplina non potesse essere altro che quello di elaborare un nuovo quadro epistemologico. Già alla fine degli anni Ottanta alcuni filologi, tra cui ricordo Raul Mordenti, Philip Brockbank, Peter Robinson e Francisco Marcós Marín, avevano cominciato a esprimere insoddisfazione per gli strumenti e le metodologie tradizionali della critica testuale, elaborando da diverse prospettive teoriche e culturali, modelli e prototipi di edizione elettronica. E nel mondo anglo-americano a partire dai primi anni Novanta si sfornano a ritmo continuo prodotti e risorse che si pongono dichiaratamente non solo oltre l'edizione critica (McGann), ma anche oltre il testo.

Pur essendo queste posizioni fra le più avanzate nel settore e pur avendo prodotto importanti risultati pratici e teorici, il processo di digitalizzazione implica traumi assai più profondi del riassetto della textual scholarship. La comunicazione digitale (di ciò stiamo parlando: perché il testo è stato costretto a cedere un po' della sua "maestà"), va oltre l'orizzonte di ricostruzione storico-critica. Il progetto che ho dedicato qualche anno fa a Valerio Magrelli è un esempio di che cosa può accadere nel momento in cui si avvia, nella cornice digitale, una qualsiasi procedura per la creazione di un oggetto di comunicazione complesso (e ciò anche al di là delle mie intenzioni iniziali). La Magrelli Genetic Machine non è infatti solo un modo di rappresentare la genesi di un testo già pubblicato, già scritto (le differenti manifestations come le chiama Shillingsburg, ma un oggetto indipendente, un nuovo inter-testo. Forse un testo che mentre si guarda, si riscrive. Si tratta forse di una 'ipotesi di lavoro', come venivano definite alcune edizioni critiche? Ma l'edizione critica era ed è ideologicamente legata alla ricostruzione dell'originale perduto o della volontà autoriale. Lì il filologo scandaglia l'origine ed elabora cronologie6, qui l'autore si liquefà nel mare delle varianti; fluttua7, come dire, su sé stesso. Se l'autore è uno, le scritture-letture sono molteplici. Quale delle due (o delle tre) è illusione? Oggi tali identità liquide vanno ben oltre l'orizzonte post-filologico e s'incarnano nelle forme di mashup ${ }^{g}$ e remix del Web 2.0, dove la nostra traccia si espande in una miriade di micro-testi alfanumerici: dai "Mi piace" di Facebook ai commenti ai blog, dai tweets alle etichette (folksonomies) con i quali descriviamo le risorse o i siti che visitiamo o archiviamo, ecc. Una miriade di para, micro e 
meta-testi che ci de-scrivono e dai e attraverso i quali siamo rappresentati (Fiormonte, "Pragmatica digitale”).

Parallelamente a questa quotidianità pervasiva abbiamo le forme "native" della scrittura digitale: spazi dove la scrittura, quella delle macchine e quella dell'uomo, si fanno azione nel tempo. Sono un esempio le rappresentazioni interattive (costa fatica chiamarli videogame) come Façade (http://www.interactivestory.net/) di Michael Mateas o le centinaia di sperimentazioni che ricadono sotto l'instabile definizione di digital literature (Hayles; Riva).9 Una delle opere che mettono in scena performativamente questa processualizzazione, Screen (http://www.noahwf.com/screen/index.html), è stata realizzata da Noah Wardrip-Fruin presso la Brown University. Su una delle tre pareti (alle spalle non c’è nulla) viene proiettato e allo stesso tempo letto da una voce un testo poetico. Dopo poco comincia l'interazione. Parole singole o frasi intere si staccano dalle pareti come una pellicola e ci vengono incontro fluttuando. Pur avendo visto e interagito con l'opera confesso che è difficile descriverne a parole l'intensità semiotica, aurale e cinetica.

Dall'altra parte abbiamo il lavoro di edizione dei testi che viene in qualche modo "illuminato" e al contempo scosso da queste forme post-testuali. Le tecnologie di produzione non sono più disgiunte da quelle di conservazione. È come se il riflesso delle forme native di comunicazione digitale si riverberasse all'indietro, colpendo al cuore l'antico sistema di "valori”: modi, sguardi, metodologie.

L’edizione critica e la sua (le sue) verità non crollano per effetto della digitalizzazione, ma perché questa ha fatto nascere forme di comunicazione, come accade ad esempio nella script act theory di Peter Shillingsburg (40-78), che "relativizzano," o meglio circoscrivono la (le) "verità" di quelle precedenti, mostrandone i limiti - il bias culturale. È il processo descritto da Innis, e poi da McLuhan, più di cinquanta anni fa.

Occorre a questo punto chiarire che quando parliamo di "limiti" del testo, non stiamo parlando soltanto di limiti intrinseci al medium o ai suoi prodotti - la letteratura. Edward Morgan Forster diceva che i limiti della letteratura sono negli occhi con cui osserviamo la realtà (Foster 165). Ma purtroppo la vista di questi "occhi nuovi” viene stroncata sul nascere. La proiezione del passato sul futuro, incarnata nel paradigma della conservazione (oggi prevalente anche nelle Digital Humanities), è dal punto di vista etico l'inverso del continuo patteggiamento al quale è sottoposto ogni atto comunicativo (ivi inclusa la lettura di un testo). Dietro la conservazione vi è lo "spirito sistematico”, al quale Giorgio Colli attribuisce il "disseccamento"10 della filosofia, che ha scelto come suoi strumenti la scrittura e oggi continua il suo dominio attraverso un'interpretazione teologico-tipografica della comunicazione digitale. Non può essere un caso che il risultato della maggiore libertà di scambio - di comunicazione - nella Rete ha prodotto legislazioni ancora più repressive di quelle del mondo della carta (vedi la European Copyright Directive o il Digital Millenium Act negli USA).

Non nego il rischio che si può correre accogliendo in modo radicale queste tesi - né l'accusa di relativismo decostruzionista. Insomma, il pericolo dell'oblio. Ma d'altra parte non serve negare la possibilità dell'oblio per poterlo sfuggire. L'oblio non si fugge, si supera. 
Attraverso forme di rappresentazione che facciano vivere (e rivivere) il passato. Perché se davvero il passato non è più in grado di parlarci, non possiamo dare la colpa al "balbettio dei [nuovi] media” o alla deperibilità dei supporti. Perché non c’è colpa nel cambiamento.

Ma è tempo di concludere lì dove abbiamo iniziato.

\section{Epilogo. Non c'è più Tempo}

La notte dell'assassinio di Fëdor Pàvlovič Karamazov, il figlio Mitja, con le mani e il volto imbrattati di sangue, corre a casa di Agrafena Aleksàndrovna (Grùšen'ka), dove, sgomento, riceve la notizia che l'amata è partita per la vicina cittadina di Mòkroe. In questi e nei successivi minuti Mitja matura la "decisione improvvisa” che lo porterà a costruire, con le sue stesse mani, il castello di accuse che ne sancirà la condanna. Mitja Karamazov, in preda alla più grande eccitazione, decide di spendere in baldoria il resto dei tremila rubli ottenuti con l'inganno da Katerina Ivànovna. Si reca dunque alla bottega di Plòtnikov e qui ordina Champagne e ogni sorta di ben di Dio, dopodiché, affittate due carrozze, una per lui e una per i viveri, corre il più veloce possibile a Mòkroe. Ma mentre nella locanda del paese si fa festa e Mitja riconquista Grùšen'ka, in città si sparge la voce dell'assassinio di Fëdor Pàvlovič. In poco tempo viene organizzata una spedizione per raggiungere e interrogare Mitja, principale sospettato. A Mòkroe giungono fra gli altri il giudice istruttore Nikolàj Parfènovič, l'ispravnik Michaìl Makaryč e il sostituto procuratore Ippolìt Kirillovič. Dostoevskij divide il serrato interrogatorio in cinque capitoli: Tribolazione I, II e III, II procuratore prende in trappola Mitja, Il grande segreto di Mitja. Le beffe. Ed è quest'ultimo quello che ci interessa. Giunti a quel punto dell'interrogatorio tutti i presenti sono convinti della colpevolezza di Mitja, ma egli crede di giocare il tutto per tutto raccontando ciò che ritiene il suo segreto più grave, ovvero la complessa dinamica del "furto" dei tremila rubli ai danni di Katerina Ivànovna. Dopo aver raccontato come aveva ottenuto il denaro, Mitja spiega che nonostante ciò che affermano molti testimoni egli non aveva speso tutti i tremila rubli, ma ne aveva conservati millecinquecento. Enumera dunque una serie di casi in cui la colpa, a suo parere, ne sarebbe risultata attenuata: per esempio nel caso avesse restituito subito metà dei soldi o se al contrario li avesse sperperati tutti immediatamente. Entrambe queste eventualità appaiono agli occhi di Mitja meno gravi di aver conservato i soldi. Tale atto gli sembra insopportabile perché denota da parte sua un calcolo, oltre che il protrarsi della sua impotenza: "è un mese intero che non riesco a decidermi, ecco! Ebbene, è una bella cosa secondo voi, è una bella cosa?” Tuttavia i giudici di fronte a queste spiegazioni rimangono freddi ("ma lasciamo da parte... queste sottigliezze"): al procuratore interessa piuttosto l'uso che il sospettato avrebbe fatto di quei millecinquecento rubli. Ed ecco allora Mitja prorompere con la sua teoria:

Ah, sì, infatti! - gridò Mitja, battendosi la fronte. - Perdonatemi, io vi faccio perdere tempo e disperare e intanto non vi spiego la cosa più importante, se no avreste capito subito, perché l'infamia è appunto nello scopo che avevo! Vedete: il vecchio, il defunto, continua a insidiare Agrafena Aleksàndrovna, e io ero geloso, allora credevo che lei fosse incerta fra lui e me. ... Allora tirai via, a malizia, metà dei tremila rubli e li cucii dentro un pezzetto di stoffa, li cucii a sangue freddo, per 
calcolo, li cucii prima di ubriacarmi, e poi, quando li avevo già cuciti, con l'altra metà andai a fare baldoria! No, è una bassezza! Avete capito ora? ...

Per tutto il tempo che ho portato addosso quei millecinquecento rubli, cuciti e attaccati al collo, tutti i giorni, a tutte le ore io mi dicevo: "Sei un ladro, sei un ladro!"

Il procuratore scoppiò in una gran risata... (Dostoevskij 986-987).

Il cuore della toccante quanto inutile contro-requisitoria di Mitja è costituito da questa confessione. Il discorso auto-accusatorio ruota intorno a alla relazione fra scopo del crimine e tempo (i giorni, le ore) lungo il quale il danno, per così dire, si estende. Egli è colpevole perché ha rubato, ma lo è di più perché ha continuato a rubare: non fu solo l'atto di rubare a costituire il danno, ma i mesi passati senza reagire. Lo scorrere della vita è colpa. In altre parole Dmitrij Karamazov enuncia il principio che l'etica è funzione del tempo. Più il tempo si dilata, più si restringe lo spazio dell'etica. Scontata la reazione negativa dall'autorità giudiziaria, che ribatte infastidita e addirittura si fa beffe delle preoccupazioni di Mitja. È qui che Dostoevskij offre una geniale rappresentazione di ciò che avviene quando la legge è chiamata a interpretare e applicare la norma in base alla realtà vissuta. L'incomprensione dei gestori del "tempo fisso" della legge si riflette e si lega all’accanita ricerca di una verità che può scaturire solo da un'analisi dei fatti e da una valutazione delle prove; per l'autorità giudiziaria è irrilevante per quanto i rubli siano stati attaccati al collo del sospettato. Tali fatti e prove però sono "staccati" dal tempo perché la legge, applicandosi a tutti casi concreti, non può che astrarre da essi e dunque esistere fuori dal tempo. Ciò che la legge non può comprendere, e anzi rifiuta o censura, non è semplicemente l'ossessione bislacca di un probabile criminale, ma la dinamicità dei processi temporali. Il tempo è in grado di generare l'etica, ma la legge può condannare solo il fatto ingabbiato nel tempo statico del suo giudizio trascendentale, fissato una volta per tutte nel testo.

L’armatura Testo-tempo-verità emerge dunque nella sua rilucente potenza: la metafisica del testo, incarnata nella filologia - come dice Segre, "il testo non ha una natura materiale" (Segre, Avviamento, 378) - opera una semplice sostituzione: dalla verità teologica a quella filologica. Non si tratta dunque di nemici ma di alleati: entrambe lavorano per la ricerca della verità e la filologia, come il fratello Diritto, si nutre di prove e indizi. Tale verità però è già data nel momento in cui si postula che da qualche parte, al di fuori del tempo vissuto, essa esista e sia indirettamente o direttamente attingibile. Nello stesso gelido empireo del tempo chiuso stanno assise la Verità e il suo prodotto, il Testo. Le scienze umanistiche sono state, finora, le principali alleate di questa visione - secondo Canfora, "solo dopo aver ricostruito il testo si dovrebbe approdare (eventualmente) a scoprire quale verità esso contenga" (Canfora 11) - come se l'operazione di ricostruzione fosse del tutto asettica.

Spero che ormai sia chiaro lo scopo di questa conclusione: ricordare come i conflitti che sorgono nella produzione, uso e gestione dei contenuti digitali non siano esclusivamente di natura economica o sociale. Dietro l'incomprensione o la difficoltà ad affrontare i problemi scatenati dalla digitalizzazione del testo vi è una colossale rimozione del tempo vivo della comunicazione. ${ }^{11}$ Bachtin parla della "interrelazione dinamica” e della "lotta" fra i due 
momenti che determinano "il testo come enunciazione: il suo progetto (intenzione) e l'attuazione di questo progetto”. Il rapporto con il senso di qualsiasi comunicazione per Bachtin è sempre dialogico:

La stessa comprensione è già dialogica... L'evento della vita del testo, cioè la sua autentica essenza, si svolge sempre sul confine tra due coscienze, tra due soggetti. ... È un incontro di due testi: di quello pronto e di quello che si crea in reazione al primo, quindi un incontro di due soggetti, di due autori. Il testo non è una cosa. (Bachtin 295; corsivo mio)

Un nuovo tempus digitale scaturisce da un duplice processo di scrittura-lettura identitaria della rete: l'interazione e dialogo con e fra le macchine (Sordi). Ma la digitalizzazione fino ad oggi è stata prevalentemente interpretata come controllo, recupero e gestione del dato: e il dato, come Mitja, può essere solo processato. Ai giudici-teologi della digitalizzazione, interessa l'uso. Trovare, gestire, controllare. E condannare. Di qui un'interpretazione prevalentemente chiusa del testo, trattato appunto come cosa, com'è negli archivi, nelle biblioteche e nei relativi motori di ricerca. L'alleanza fra mondo teofilologico e logica del computer (il processore come "agente onniscente isolato") è stata sin qui salda. Ma nell'immersività simbiotica di esperimenti come il citato Screen, come nell'uso quotidiano dei social media, il testo sfugge, "si evolve cooperativamente," così come ci insegna l'etnografia (Tyler), e noi scriviamo e leggiamo insieme alle macchine, lasciando tracce che vengono surrogate da altri, manipolando e rifondendo contenuti (ma gli altri chi o cosa?). L'autorialità collettiva, la co-autorialità delle macchine, non sono più solo una simulazione. E la risata del procuratore Parfènovič, benché ancora riecheggi sinistra nei corridoi dove si decidono i destini e i contorni delle nostre identità, è destinata a spegnersi “nell'assordante sovrapposizione di voci e parole” della Rete.

\footnotetext{
${ }^{1} \mathrm{Vi}$ sono alcune persone che hanno visto nascere questo scritto e senza le quali non avrei mai avuto il coraggio di dargli forma. Le ringrazio tutte, ma un pensiero particolare va a B.R. Infine, mi sia permesso ricordare il Maestro Vedanta Ramesh Balsekar, scomparso a Bombay il 29 settembre 2009 all'età di 92 anni. Tuja Namo (Mi inchino a te). Una versione precedente del contributo è apparsa in Fiormonte, "Tiempo Texto Verdad."

${ }^{2}$ Il contesto storico del passo biblico è la divisione in satrapie a opera di Dario I di Persia nel 521 a.C.

${ }^{3}$ La precettistica segriana (il suo sembra un manuale per novizi: "guai a guastare la purezza" ecc.) è il risultato pratico del processo sociale e storico che vede la stabilizzazione del testo come base per la moderna religione del prodotto. Non è un caso che nel più ambizioso e influente progetto educativo occidentale mai concepito (e in parte realizzato), quello di Wilhelm von Humboldt, la filologia avrebbe sostituito la teologia (Vega 265-266).

${ }^{4} \mathrm{Si}$ veda il filone della semiotica retorico-ermeneutica, incarnato da François Rastier, il quale critica la "concezione metafisica" del testo della linguistica: "Al di là della complessità che caratterizza la testualità in quanto tale, infatti, i testi utilizzano non soltanto lingue, generi e stili ma anche sistemi grafici e tipografici.... La semiotica ha in genere trascurato tutti questi aspetti, al pari dei linguisti che si limitano allo studio della morfosintassi.” (Rastier 110).
} 
${ }^{5}$ Vedi la sezione dedicata a Magrelli su: <http://www.selc.ed.ac.uk/italian/digitalvariants/>

6 “Cosicché, attraverso l'ingenuità delle cronologie, si verrebbe fatalmente ricondotti verso un punto sempre più remoto, mai presente in nessuna storia; questo punto non sarebbe altro che il proprio vuoto; e a partire da esso tutti gli inizi non potrebbero essere altro che delle riprese o delle occultazioni .... Non bisogna rimandare il discorso alla lontana presenza dell'origine; bisogna affrontarlo nel meccanismo della sua istanza" (Foucault 34-35).

${ }^{7}$ Uno degli strumenti di visualizzazione disponibili sul sito è stato battezzato infatti floating variants.

${ }^{8}$ Si veda l'esperimento realizzato da Paolo Sordi, "Io sono", un sito che si alimenta esclusivamente dei contenuti generati dai social media usati dall'autore:

http://iosono.infolet.it.

${ }^{9}$ Vedi anche l'antologia disponibile su <http://www.hermeneia.net/eng/espais/literatura.html >.

10 "Così nasce la filosofia, creatura troppo composita e mediata per racchiudere in sé nuove possibilità di vita ascendente. Le spegne la scrittura, essenziale a questa nascita. E l'emozionalità, a un tempo dialettica e retorica, che ancora vibra in Platone, è destinata a disseccarsi in un breve volgere di tempo, a sedimentarsi nello spirito sistematico" (Colli 116).

${ }^{11}$ Tale incomprensione si ricollega alla teoria standard della comunicazione, che descrivendo la comunicazione come un "passaggio," scambia il "corpo morto" della comunicazione, l’oggetto, per il suo corpo vivente, "la conversazione” (Ronchi 193-198).

\section{Works Cited}

Antonelli, Roberto. "Spazio, tempo e testualità: l'ecdotica tra regioni e nazione." Testi $e$ tradizioni. Le prospettive della filologia. Atti del seminario, Alghero, June 7 2003. Ed. Paolo Manichedda. Cagliari: Centro di Studi Filologici Sardi, 2004, 17-32. Print

Bakhtin, Mijail Mijaïlovich. L'autore e l'eroe: teoria letteraria e scienze umane. Torino: Einaudi, 1988. Print

Barbaglia, Silvio. "La rilevanza ermeneutica delle disposizioni canoniche dei testi nelle Sacre Scritture. Metodo ed esemplificazioni." Il testo biblico in tensione fra fissità canonica e mobilità storica. Atti dell'XI Convegno di Studi Veterotestamentari (Torreglia, September 6-8, 1999). Ed. Silvio Barbaglia. Bologna: Edizioni Dehoniane Bologna / Ricerche Storico Bibliche, 2001. 185-268. Print.

Benozzo, Francesco. “Etnofilologia.” Ecdotica 4 (2007): 208-230. Print.

Bologna, Corrado. "Per una filologia degli scarti, dei dislivelli, delle fratture”. Testi $e$ tradizioni. Le prospettive della filologia. Ed. Paolo Manichedda. 49-79. Print.

Canfora, Luciano. Filologia e libertà. La più eversiva delle discipline, l'indipendenza di pensiero e il diritto alla verità. Milano: Rizzoli, 2008. Print.

Colli, Giorgio. La nascita della filosofia. Milano: Adelphi, 1975. Print.

Dostoevskij, Fëdor. I fratelli Karamazov. Tutti i Romanzi. Trans. Pina Maiani, Vol. II. Firenze: Sansoni, 1988. Print. 
Fiormonte, Domenico. "Pragmatica digitale. Paratesti, microtesti e <metatesti> nel web." Testi brevi. Atti del convegno internazionale di studi, Università Roma Tre, 8-10 giugno 2006. Eds. Maurizio Dardano, Gianluca Frenguelli, Elisa De Roberto. Roma: Aracne, 2008. 65-84. Print.

---. “Tiempo Texto Verdad”. Maestra en mucho. Estudios filológicos en homenaje a Carmen Isasi Martínez. Eds. Sara Gómez Seibane, José Luis Ramírez Luengo. Buenos Aires: Voces del Sur, 2011. 79-91. Print.

Forster, Edward M. Aspetti del romanzo. Trad. Corrado Pavolini. Milano: Garzanti, 1991. Print.

Foucault, Michel. L'archeologia del sapere. Trad. di Giovanni Bogliolo. Milano: Rizzoli, 1980. Print.

Hayles, N. Katherine. Electronic literature: new horizons for the literary. Notre Dame (Ind.): University of Notre Dame Press, 2008. Print.

Innis, Harold. Impero e comunicazioni. Roma: Meltemi, 2001. Print.

Jaynes, Julian. Il crollo della mente bicamerale e l'origine della coscienza, Milano: Adelphi, 1984. Print.

Liverani, Mario. Antico Oriente. Storia, società, economia. Roma-Bari: Laterza, 2005. Print.

---. Oltre la Bibbia. Roma-Bari: Laterza, 2003. Print.

Lucía Megías, José Manuel. “La 'Informática Humanística’: notas volanderas desde el ámbito hispánico.” Incipit 23 (2003): 91-114. Print.

Manichedda, Paolo, ed. Testi e tradizioni. Le prospettive della filologia. Atti del seminario, Alghero, June 7 2003. Cagliari: Centro di Studi Filologici Sardi. Print.

McGann, Jerome J. Radiant Textuality. Literature after the World Wide Web. New York: Palgrave MacMillan, 2001. Print.

Mordenti, Raul. L'altra critica. La nuova critica della letteratura fra studi culturali, didattica e informatica. Roma: Meltemi, 2007. Print

Numerico, Teresa. Alan Turing e l'intelligenza delle macchine. Milano: Franco Angeli, 2005. Print.

Prato, Gianluigi. "Gli scritti biblici tra utopia del canone fisso e fluidità del testo storico." Ed. Domenico Fiormonte. Canoni liquidi. Variazione culturale e stabilità testuale dalla Bibbia a Internet. Napoli: ScriptaWeb, 2011. Print.

Reboul, Olivier. Introduzione alla retorica. Bologna: Il Mulino, 1996. Print.

Riva, Massimo. Il futuro della letteratura. L'opera letteraria nell'epoca della sua (ri)producibilità digitale. Napoli: ScriptaWeb, 2011. Print.

Ronchi, Rocco. Filosofia della comunicazione. Torino: Bollati Boringhieri, 2008. Print.

Segre, Cesare. Avviamento all'analisi del testo letterario. Torino: Einaudi, 1985. Print. 
---. Ritorno alla critica. Torino: Einaudi, 2001. Print.

Shillingsburg, Peter. From Gutenberg to Google. Electronic Representations of Literary Texts. Cambridge: Cambridge University Press, 2006. Print.

Sordi, Paolo. "Noi (u)siamo la macchina. Dal web design all'etnografia digitale.” Lezione al corso di laurea magistrale "Forme e generi della testualità digitale", Università Roma Tre, 26 maggio 2009. <http://www.slideshare.net/psordi/noi-usiamo-la-macchina >. Web.

Storey, H. Wayne. “All'interno della poetica grafico-visiva di Petrarca.” Rerum Vulgarium Fragmenta. Cod. Vat. Lat. 3195. Commentario all'edizione in fac-simile. Eds. Gino Belloni, Furio Brugnolo, H. Wayne Storey, Stefano Zamponi. Roma-Padova: Editrice Antenore, 2004. 131-171. Print.

Tyler, Stephen A. "Post-Modern Ethnography: From Document of the Occult to Occult Document." Writing Cultures: Poetics and Politics of Ethnography. Eds. James Clifford, George M. Marcus. Berkeley: University of California Press, 1986. 122-140. Print.

Vega, María José. "El laboratório filológico”. Review of Pascale Hummel. Histoire de l'Histoire de la Philologie. Étude d'un genre épistémologique et bibliographique, Gèneve, Droz, 2000. Ecdotica 1 (2004): 255-266. Print.

Wardrip-Fruin, Noah. “Screen.” Leonardo 39, 2 (2006): 103-132. Print.

Woodmansee, Martha. The author, art, and the market. Rereading the history of aesthetics. New York: Columbia University Press, 1994. Print. 